\title{
INDIRECT ORTHODONTIC BONDING WITH DROP-SHAPED HOT GLUE TRAY: A SIMPLE AND COST-EFFECTIVE TECHNIQUE
}

\author{
Carolina Ribeiro STARLING ${ }^{1}$ (D), Lílian Siqueira de LIMA $^{1}$ iD, Marcos Alan Vieira BITTENCOURT² iD
}

\footnotetext{
${ }^{1}$ Postgraduate Program in Orthodontics, Department of Pediatric Dentistry and Orthodontics, Federal University of Rio de Janeiro, Rio de Janeiro, Rio de Janeiro, Brazil.

2 Department of Pediatric and Community Dentistry, Federal University of Bahia, Salvador, Bahia, Brazil.
}

Corresponding author:

Marcos Alan Vieira Bittencourt

Email: alan_orto@yahoo.com.br

How to cite: STARLING, C.R., LIMA, L.S. and BITTENCOURT, M.A.V. Indirect orthodontic bonding with drop-shaped hot glue tray: a simple and cost-effective technique. Bioscience Journal. 2021, 37, e37088. https://doi.org/10.14393/BJ-v37n0a2021-59299

\begin{abstract}
Despite being associated with more accuracy, the indirect bonding procedure is not yet the gold standard, probably because of sensitivity of the numerous variables that must be controlled. The aim of this article was to present a modified, standardized, and low-cost indirect bonding technique that allows this procedure to be performed successfully. The technique covers an initial clinical stage, to obtain the models; a laboratory stage, which involves placement of brackets on the models following the facial axis of the clinical crown and the labial projection of the marginal ridges of the posterior teeth and construction of transfer tray using hot glue; and a second clinical stage, to properly transfer the brackets to patient's teeth. Hot glue used to build the tray molds the teeth and perfectly adapts to the teeth, having enough stiffness to maintain their anatomy and the position of the brackets, but also presenting adequate flexibility to allow removal of the tray avoiding excessive stress over the brackets. In conclusion, the new simplified indirect bonding technique presented here provides a precise placement of brackets on the models, a cheaper way to transfer them to patient, and an easy removal of transfer tray, being a very simple and cost-effective method.
\end{abstract}

Keywords: Corrective Orthodontics. Dental Bonding. Orthodontic Adhesives.

\section{Introduction}

Regardless of its complexity, one of the keys to an efficient orthodontic treatment relies on correct positioning of brackets during bonding. Although less popular, the indirect bonding technique usually provides better vision and enough time to place brackets on the models, facilitating this procedure to some degree and, as a result, being associated with more accuracy (Nawrocka and Lukomska-Szymanska 2020). First described in detail as a concept in 1972, by Silverman et al. (1972), indirect bonding emerged in the same decade of pre-adjusted appliances and light-cured composites, but orthodontists were not immediately seduced due to some difficulties existing at that time. The tray was opaque, silicone made, preventing the use of primers and light-curable resins, and had a shape that made it difficult to remove excess resin before it hardened, requiring an additional step of removing the excesses at the end of the bonding. Furthermore, brackets were bonded to models with washable white glue, and spaces between dental surfaces and bracket bases could produce displacements during bonding since resin was delivered at the bracket bases just before transfer to the patient's teeth.

Shortly after, these disadvantages began to be overcome. The customization of bracket bases, proposed by Thomas (1979), became the foundation for contemporary indirect bonding. They used composite at the bracket base before being bonded to the model, providing a copy of the buccal surface of 
the teeth for posterior use of a two-part liquid sealant to bond the brackets to the dentition. Later, the use of transparent trays (Read and O' Brien 1990) allowed the application of light-cured adhesive resins and the visual confirmation of ideal tray placement and bracket positions. This visibility also allowed the orthodontist to clean off excess composite around the brackets and apply light cure when fully satisfied with bracket position and hygiene, promoting less plaque accumulation during treatment (Kalange 2004; Zanini 2016; Aggarwal and Aggarwal 2018). Additionally, the various advantages of indirect technique have remained, especially the better visualization of teeth in the model than in patient's mouth, offering greater precision in bracket positioning (Guenthner and Larson 2007; Agarwal and Kulshrestha 2017; Singh et al. 2019) and improving ability to bond posterior teeth (Aggarwal and Aggarwal 2018; Kalra et al. 2018). Literature also mentions the reduced chair time (Kalange 2004; Pamukçu and Özsoy 2016; Li et al. 2019; Singh et al. 2019) and the possibility of simultaneous bonding of several accessories (Kalange 2004; Aggarwal and Aggarwal 2018), so making the procedure attractive to both operator and patient.

Some variations have been described to carry out this technique over the years with several advances, standing out especially on the adhesive used to attach brackets to casts (Sondhi 2007) and on the transfer tray material used, eg, thermal glue (White 1999), rapid prototyped (Ciuffolo et al. 2006), silicone (Guenthner and Larson 2007), vacuum-formed (Sondhi 2007), vinil polysiloxane (Grünheid et al. 2016), or associated methods (Ciuffolo et al. 2012). Recently, great emphasis has been placed on digital indirect bonding (Christensen and Cope 2018; Spitz et al. 2018; Layman 2019; Balut et al. 2020), bracket placement being controlled digitally, and offering very promising results (Kim et al. 2018; Duarte et al. 2020). The high costs involved in this process, however, still do not allow its routine use among most orthodontists. Despite the availability of these proposed variations, the indirect procedure is not yet the gold standard, probably because of numerous procedural variables that must be controlled to obtain successful indirect bonding (Demirovic et al. 2018). It is supported by the extra expenses and duration of laboratory phase, and sensitivity of the multiphase technique. Then, the aim of this article was to present a modified, standardized, and low-cost indirect bonding technique for routine and effective use of the indirect bonding procedure.

\section{Indirect Bonding Technique}

1. Perform dental prophylaxis and take accurate impressions of both arches using high quality alginate, following manufacturer's instructions. Check the impressions in order to avoid potential flaws.

2. Use hard dental stone to make the casts as to obtain greater resistance and fidelity. After they are dried, clean them and remove all defects and bubbles from the teeth, with great care so that tooth anatomy should not be altered.

3. Make bracket positioning guidelines on the models (Figure $1 \mathrm{~A}-\mathrm{C}$ ), using a black pencil, as follows:

a. Mark the cervical contour of all teeth, highlighting the line between tooth and gingival margin, and the central groove of occlusal surfaces of posterior teeth, showing possible rotations.

b. Mark the buccal projection of the marginal ridges of premolars and molars with a line connecting mesial to distal ridges of each tooth.

c. Mark the long axis of each tooth on the center of its clinical crown, using the patient's panoramic radiograph as an auxiliary method to visualize tooth angulations and avoid possible mistakes, increasing accuracy.

d. Using a Boone bracket position gauge (3M/Unitek ${ }^{\mathrm{TM}}$, Monrovia, CA, USA) and having the clinical crown height of the second premolar as reference check the possible bracket slot positions. Then, after defining the height to be used, mark, using the gauge and the pencil, the slot heights on the incisors, canines, and one first premolar, with a line perpendicular to the long axis on incisors and canines, and parallel to the line of the marginal ridge on the first premolar.

e. Measure the distance between the two horizontal lines marked on the first premolar, using a caliper or a bow divider, and reproduce it on the remaining posterior teeth. Doing this, the anterior teeth will be connected with the posterior ones through the first premolar, and all posterior teeth will be connected to each other by the heights of the marginal ridges, so that they will be leveled at the end of orthodontic treatment. 
f. Review treatment plan with casts in occlusion, and brackets previously selected prior to mark the guidelines on the lower cast, so as to avoid setbacks during definitive bonding, such as lower brackets interfering in post bonding occlusion.

4. Apply a thin layer of separating medium (Cel-Lac, SS White, Rio de Janeiro, RJ, Brazil) on the tooth surfaces with a brush and wait a few minutes to permit the cast to dry fully.

5. Add a light-curable composite adhesive (Transbond $X T^{\mathrm{TM}}, 3 \mathrm{M}$ Unitek, Monrovia, $\mathrm{CA}, \mathrm{USA}$ ) at the bracket base, press it firmly on the cast surface, and remove any excess using fine instruments. Position each bracket following the previously established guidelines, so that its slot and axis lie over the marked lines at the clinical crown (Figure 1D). Once the positioning on the full arch is finished, use a light-curing unit (Ortholux LED, 3M Unitek, Monrovia, CA, USA) to cure the adhesive of each bracket, following the manufacturer's instructions.

6. Trays are recommended to be performed in three segments, one at the anterior region (canine to canine) and two at the posterior ones (premolars and molars), as follows:

a. With the glue gun heated, pour molten glue over the slot and wings of all brackets, extending it over the buccal surface towards the incisal edge or occlusal surface of each tooth (Figure 2A).

b. Place the molten glue on the incisal edges or occlusal surfaces of all teeth in each segment, joining it to the buccal portion already made (Figure 2B).

c. Extend the molten glue over the lingual surfaces of all teeth in each segment, completing the shape of the trays (Figure $2 \mathrm{C}$ ).

d. If necessary, increase the thickness of each segment. The final appearance of the tray can be seen in Figure 2D.

7. After the glue hardens, soak the model in water for five minutes to dissolve the separator and then gently press each bracket to dislodge it from the cast. Clean the residual stone and separating agent of adhesive bases with $70 \%$ alcohol, and then rinse and dry them fully. Trays can be used immediately or be stored in a dry environment, at room temperature and protected from light (Figure 3A).

8. The last stage of technique is the clinical bonding. Commonly recommended clinical procedures include the following steps:

a. Perform prophylaxis using oil-free and fluoride-free paste or extra-fine pumice, and etch tooth surfaces to be bonded with $37 \%$ phosphoric acid gel for 20 seconds, after proper isolation with cotton rolls. If special conditions are present, such as porcelain, composite or amalgam restorations, perform proper conditioning of the surfaces to be bonded, according to each situation, in a similar way to direct bonding. Rinse for additional 20 seconds and, after new isolation, dry fully.

b. Apply a thin layer of primer (Transbond $\mathrm{XT}^{\mathrm{TM}}, 3 \mathrm{M}$ Unitek, Monrovia, CA, USA) on the teeth of the first segment to be bonded and on their respective adhesive bases, followed by gentle air spray.

c. Carefully seat the transfer tray over teeth visually checking its proper adaptation (Figure 3B). Maintain two fingers to stabilize the tray on the teeth and other one to keep the brackets and the tooth surfaces matched. Light-cure the distal edge of the first bracket of the tray on one side for 10 seconds, then slide the tip of the light-curing unit to the next interproximal edge and light-cure also for 10 seconds, and so on until the last interproximal edge is light-cured. Move the tip to the cervical edge of all brackets, and after to the incisal/occlusal edge, and light-cure each edge for additional five seconds.

d. Remove the transfer tray with caution, starting with the glue drops on the brackets, with a tip instrument (Figure $3 \mathrm{C}$ ), until the entire tray is detached. If any bracket is accidentally debonded, cut the portion of the tray corresponding to the tooth, apply a new layer of primer on the tooth and on the customized adhesive base, seat the individual tray segment over the tooth, and bond the bracket again.

e. Repeat the same procedures for the other two tray segments.

f. Remove cotton roll isolation and floss interproximal areas to check they are open or to remove any primer flash. Immediately afterwards, archwires can be engaged (Figure 3D). 

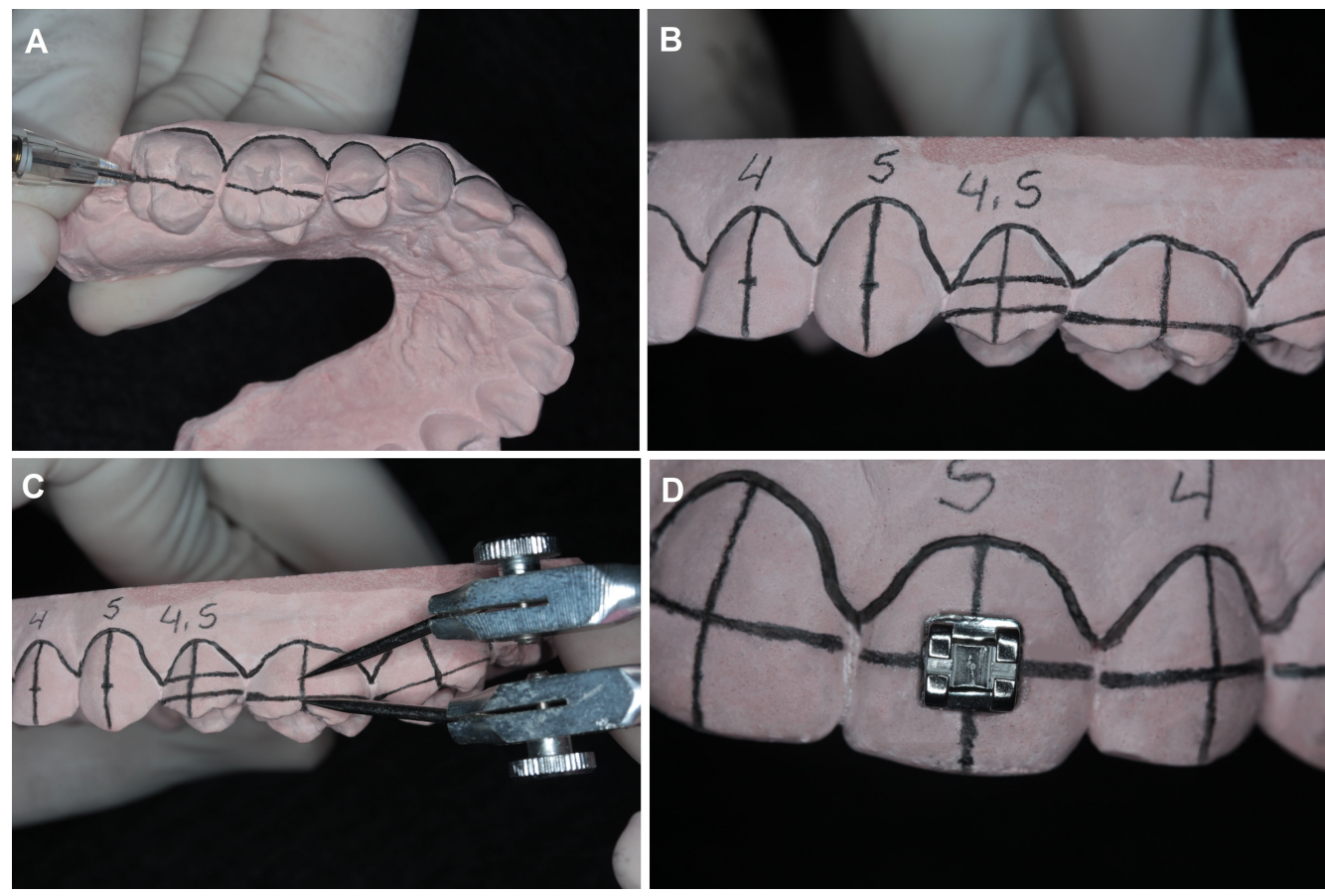

Figure 1. Reference lines drawn on cast. A - initially, cervical contour of all teeth and central groove of posterior teeth; B - marginal ridge heights were marked on posterior teeth, followed by the long axis of all teeth. Using a position gauge, slot heights on incisors, canine, and premolar were also marked. A second line, parallel to the line of the marginal ridge, was drawn on the premolar; $\mathrm{C}$ - distance between the two horizontal lines on the premolar should be measured and transferred to all posterior teeth; D - positioning of the bracket with light-curing adhesive, according to the reference lines, respecting slot height and long axis.
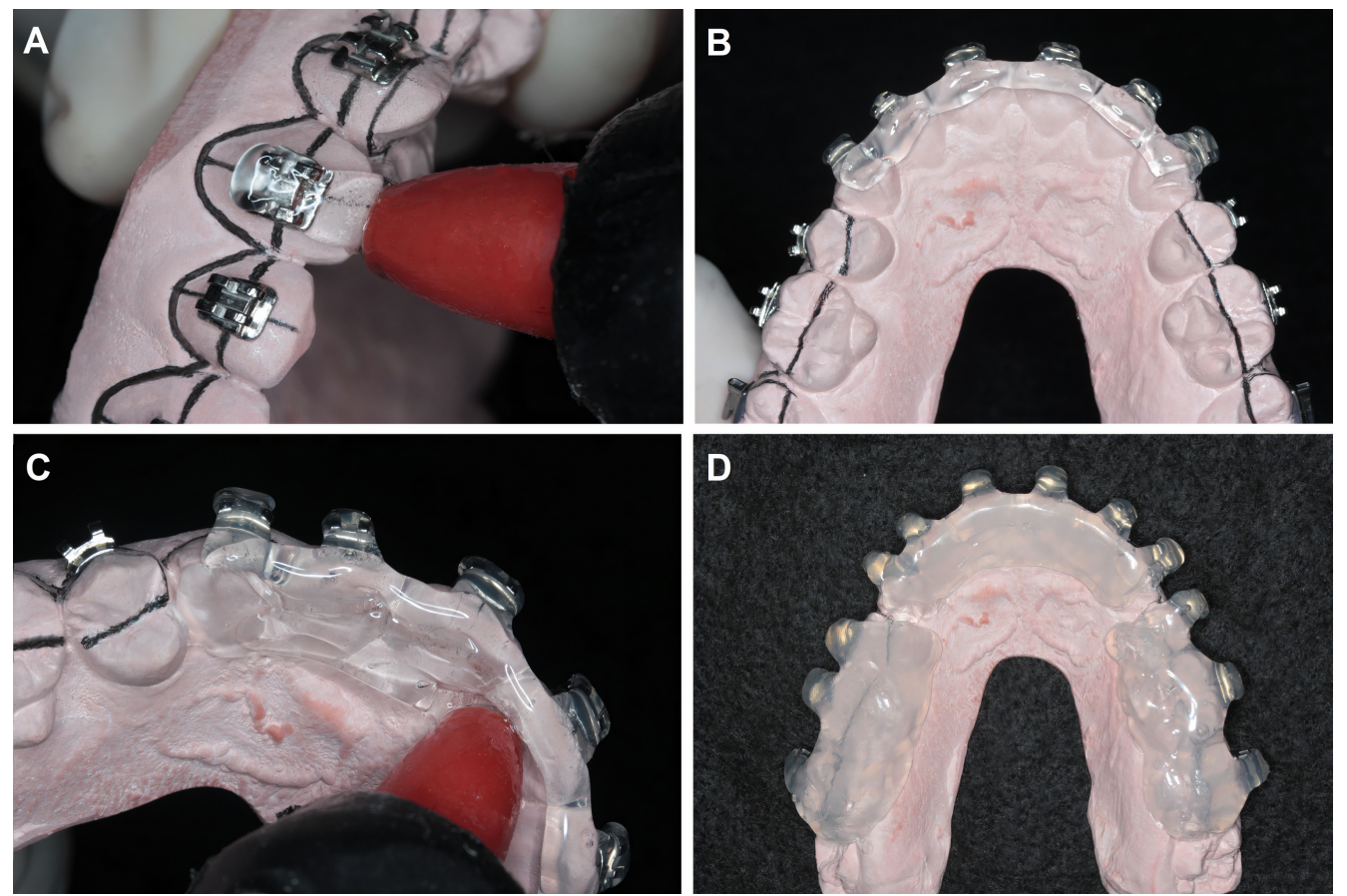

Figure 2. Creating the transfer tray. A - starting with the canine, application of molten glue over the slot and wings of bracket, extending it over the buccal surface towards the cusp; B-molten glue placed on the incisal edge of all anterior teeth; $\mathrm{C}$ - completion of the tray extending the molten glue over the lingual surface of all teeth; D - final appearance of the transfer trays. 

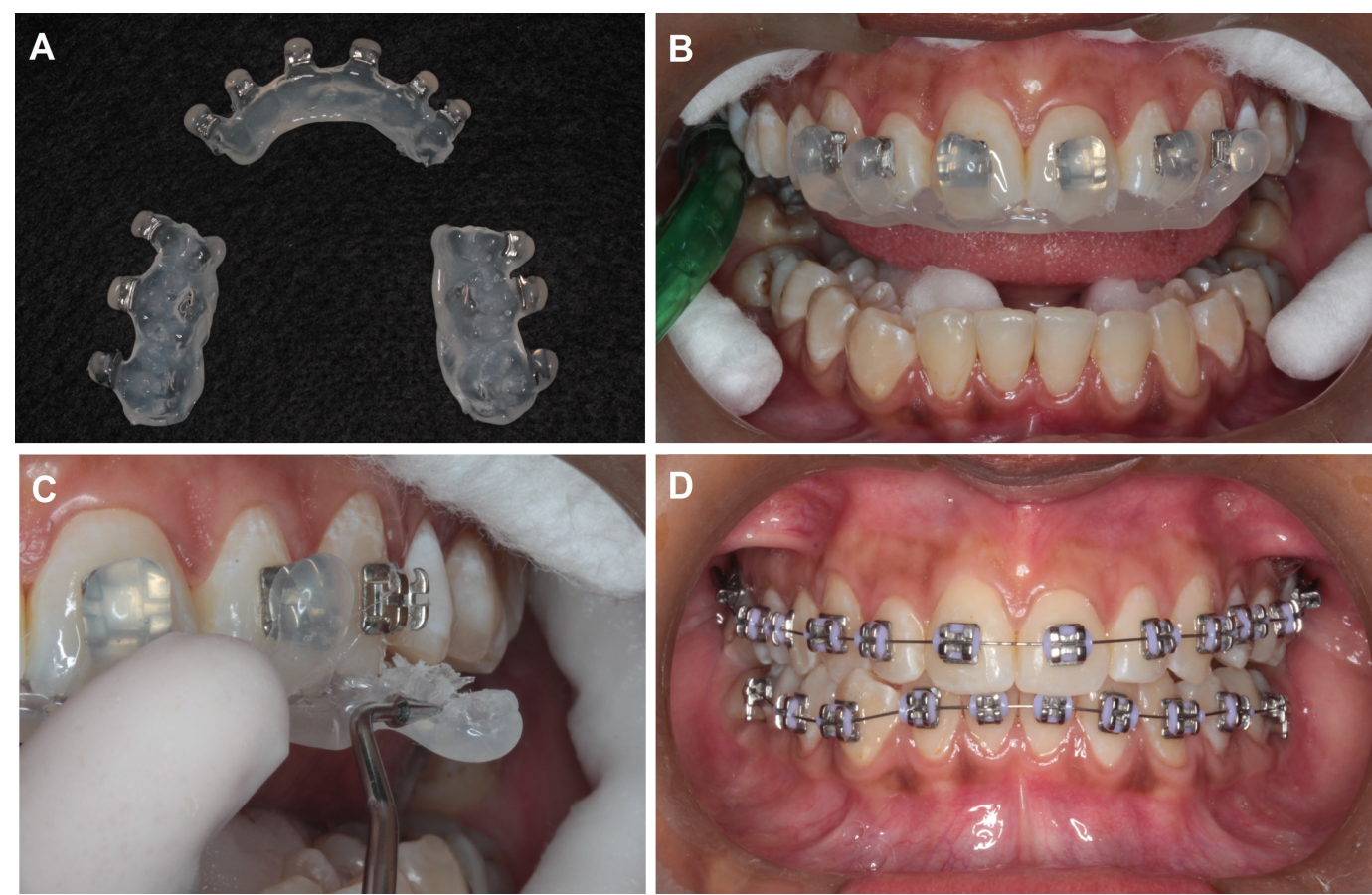

Figure 3. A - transfer tray performed in three segments, with brackets; B - transfer tray seated properly in patient's anterior teeth, ready to be light-cured; C - removal of transfer tray, starting with the drops, one at a time; $\mathrm{D}$ - end of the procedure. Orthodontic archwires can be immediately engaged into slots.

\section{Discussion}

An effective predictable bonding technique simplifies clinical operations. The indirect bonding technique should provide high accuracy in bracket placement and be of simple execution (Guenthner and Larson 2007). Achieving success with the described technique is not complex, provided attention is paid to the recommended details. It allows precise orthodontic appliance installation in only one appointment and can be used to place any bracket system commercially available. To have a reliable procedure, all variables that might influence the technique must be controlled. Impressions and models must be accurate, brackets must be precisely positioned and transfer trays properly fabricated. Hot glue is a molten polymer of ethylene vinyl acetate, approved by the Food and Drug Administration (FDA) as noncarcinogenic, nontoxic, and dimensionally stable in its solid form (White 1999).

The bracket-positioning guide performed on the models followed the facial axis of the clinical crown and the labial projection of the marginal ridges of the posterior teeth, as adopted by Ciuffolo et al. (2012). This method means a reasonable amount of in-office time to have a clinically precise bracket position on the models but allows properly aligning the teeth and leveling the arch with the marginal ridges (Kalange 2004).

Clinical experiences and experimental studies have shown that Transbond $\mathrm{XT}^{\mathrm{TM}}$ system provides excellent results when associated to indirect bonding technique (Nojima et al. 2015; Bolya et al. 2017). Authors have reported this system is very effective in tensile and shear bond strength and failure rate, when compared to other adhesives (Shimizu et al. 2012). In addition, Transbond XT ${ }^{\mathrm{TM}}$ presents similar behavior when used in direct or indirect bonding techniques, being able of reaching the same clinical efficiency when the indirect bonding technique is performed properly (Menini et al. 2014; Tessore et al. 2017; Demirovic et al. 2018; Sharma et al. 2018). The custom base adapts so closely to the tooth that it is only necessary to use a thin film of primer between the custom base resin and the etched enamel.

Another critical point concerning the indirect bonding method is transferring brackets to teeth with precision, circumstance influenced mainly by material and method of building up the transfer tray (Möhlhenrich et al. 2020). The transfer tray must have two main specific features, stability in the positioning and ease in the removing (Ciuffolo et al. 2012). Hot fluid glue molds the teeth and perfectly adapts to the patient's malocclusion, regardless the position of the teeth. The drop-shaped glue tray, described in this article, has enough stiffness to maintain the anatomy of the teeth and the position of the brackets and adequate flexibility to allow removal of the tray, standing out in a drop-wise manner, avoiding excessive force on the bracket at the time of its removal. According to Wendl et al. (2008), problems with bonding can 
arise from stress caused to the adhesive interface during transfer tray removal. Flexibility of this material prevents excessive forces over brackets while the adhesive has not yet reached its peak bond strength. This relative mechanical retention occurs regardless of the bracket's size or shape. The thermoplastic trays, on the other hand, are not recommended to be used with small sized or poor wing retention brackets (White 1999). In addition, there is no need to place ligatures or wax on each bracket to prevent penetration of tray material into the slots, as recommended by Aileni et al. (2012).

Fabrication of tray incorporating only the incisal and occlusal wings of brackets helps in direct curing of composite gingivally during bonding. A firm hold on occlusal and labial aspects of the transfer tray is necessary while curing to overcome the flexible nature of the transfer tray (Aileni et al. 2012). Similar to other techniques, the use of either single tray for entire arch or sectional trays depends on the control of isolation, and amount of crowding and imbrications of the teeth. Although the use of single tray only reduces the clinical chair side time, it is sometimes difficult to ensure a proper isolation of the entire arch, especially if bonding at the lingual surface of teeth (Paul et al. 2017). In this situation, Pamukçu et al. (2018) recommend the division of the tray into two or three pieces for maximum control during polymerization.

Currently, new perspectives on the refining of protocols to bonding processes have been indicated, especially by the incorporation of digital technology. The assorted commercial software systems are multifunctional and generally integrate three-dimensional digital models, setup and virtual bonding procedures, and prototyped transfer trays. However, Oliveira and Pretti (2018) state that, in computer-aided bonding, results diverge and seem to depend especially on the type of software system applied. Although virtual indirect bonding has been considered more accurate when compared to direct bonding (Oliveira et al. 2019), according to Israel et al. (2011), virtual bracket placement methods do not establish more precise positioning in comparison to traditional indirect bonding techniques. In addition, raising costs of equipments hinder these laboratory procedures to be carried out in a clinical office (Czolgosz et al. 2020). On the other hand, the indirect bonding technique described here is simpler, less expensive, efficient, comfortable, and it can be easily performed in the orthodontic office.

\section{Conclusions}

Indirect bonding technique is considered to be useful and efficient approach that improves the clinically efficiency of accurate bracket positioning and decreases the chair side time. The technique described in this article provides a precise placement of brackets on the cast, which is the main advantage of indirect bonding method, an easy removal of excess resin flash, a cheaper way to transfer them to patient's teeth, and an easy removal of transfer tray. It is a very simple and cost-effective method. Success to this technique requires attention to detail but does not require excessive complexity.

Authors' Contributions: STARLING, C.R.: conception and design, analysis and interpretation of data, drafting the article, and critical review of important intellectual content; LIMA, L.S.: conception and design, analysis and interpretation of data, and critical review of important intellectual content; BITTENCOURT, M.A.V.: conception and design, drafting the article, and critical review of important intellectual content. All authors have read and approved the final version of the manuscript.

Conflicts of Interest: The authors declare no conflicts of interest.

Ethics Approval: Not applicable.

Acknowledgments: Not applicable.

\section{References}

AGARWAL, P. and KULSHRESTHA, R. Comparison of accuracy of bracket placement between direct and indirect bonding techniques: an in-vivo study. Indian Journal of Orthodontics and Dentofacial Research. 2017, 3(2), 107-113. https://doi.org/10.18231/2455-6785.2017.0022

AGGARWAL, P. and AGGARWAL, R. Indirect bonding procedures in orthodontics: a review. Journal of Dentistry and Dental Medicine. 2018, 1(4), 120. https://doi.org/10.31021/jddm.20181120

AILENI, K.R., et al. Gum and gun: a new indirect bonding technique. The Journal of Indian Orthodontic Society. 2012, 46(4), $287-291$.

BALUT, N., et al. Digital orthodontic indirect bonding systems: a new wave. APOS Trends in Orthodontics. 2020, 10(3), $195-200$.

https://doi.org/10.25259/APOS 182020 
BOLYA, P., et al. Evaluation of bond strength of brackets using various indirect bonding techniques. Journal of Indian Orthodontic Society. 2017, 51(1), 15-21. https://doi.org/10.4103/0301-5742.199248

CHRISTENSEN, L.R. and COPE, J.B. Digital technology for indirect bonding. Seminars in Orthodontics. 2018, 24(4), 451-460. https://doi.org/10.1053/i.sodo.2018.10.009

CIUFFOLO, F., et al. Rapid prototyping: a new method of preparing trays for indirect bonding. American Journal of Orthodontics and Dentofacial Orthopedics. 2006, 129(1), 75-77. https://doi.org/10.1016/j.ajodo.2005.10.005

CIUFFOLO, F., TENISCI, N. and POLLUTRI, L. Modified bonding technique for a standardized and effective indirect bonding procedure. American Journal of Orthodontics and Dentofacial Orthopedics. 2012, 141(4), 504-509. https://doi.org/10.1016/i.ajodo.2010.09.035

CZOLGOSZ, I., CATTANEO, P.M. and CORNELIS, M.A. Computer-aided indirect bonding versus traditional direct bonding of orthodontic brackets: bonding time, immediate bonding failures, and cost-minimization. A randomized controlled trial. European Journal of Orthodontics. 2021, 43, 144-151. https://doi.org/10.1093/ejo/cjaa045

DEMIROVIC, K., et al. Comparison of shear bond strength of orthodontic brackets using direct and indirect bonding methods in vitro and in vivo. Acta Informatica Medica. 2018, 26(2), 125-129. https://doi.org/10.5455/aim.2018.26.125-129

DUARTE, M.E.A., et al. Reproducibility of digital indirect bonding technique using three-dimensional (3D) models and 3D-printed transfer trays. The Angle Orthodontist. 2020, 90(1), 92-99. https://doi.org/10.2319/030919-176.1

GRÜNHEID, T., LEE, M.S. and LARSON, B.E. Transfer accuracy of vinyl polysiloxane trays for indirect bonding. The Angle Orthodontist. 2016, 86(3), 468-474. https://doi.org/10.2319/042415-279.1

GUENTHNER, T.A. and LARSON, B.E. Indirect bonding: a technique for precision and efficiency. Seminars in Orthodontics. 2007, 13(1), 58-63. https://doi.org/10.1053/j.sodo.2006.11.007

ISRAEL, M., et al. A comparison of traditional and computer-aided bracket placement methods. The Angle Orthodontist. 2011, 81(5), 828-835. https://doi.org/10.2319/072110-425.1

KALANGE, J.T. Indirect bonding: a comprehensive review of the advantages. World Journal of Orthodontics. 2004, 5(4), $301-307$.

KALRA, R.K., et al. Comparison of accuracy of bracket placement by direct and indirect bonding techniques using digital processing: an in-vitro study. Journal of Clinical and Diagnostic Research. 2018, 12(9), 7-11. https://doi.org/10.7860/JCDR/2018/32466.11975

KIM, J., CHUN, Y. and KIM, M. Accuracy of bracket positions with a CAD/CAM indirect bonding system in posterior teeth with different cusp heights. American Journal of Orthodontics and Dentofacial Orthopedics. 2018, 153(2), 298-307. https://doi.org/10.1016/j.ajodo.2017.06.017

LAYMAN, B. Digital bracket placement for indirect bonding. Journal of Clinical Orthodontics. 2019, 53(7), 387-396.

LI, Y., et al. Effectiveness, efficiency and adverse effects of using direct or indirect bonding technique in orthodontic patients: a systematic review and meta-analysis. BMC Oral Health. 2019, 19(1), 137. https://doi.org/10.1186/s12903-019-0831-4

MENINI, A., et al. A 15-month evaluation of bond failures of orthodontic brackets bonded with direct versus indirect bonding technique: a clinical trial. Progress in Orthodontics. 2014, 15(1), 70. https://doi.org/10.1186/s40510-014-0070-9

MÖHLHENRICH, S.C., et al. Three-dimensional evaluation of bracket placement accuracy and excess bonding adhesive depending on indirect bonding technique and bracket geometry: an in-vitro study. Head and Face Medicine. 2020, 16(1), 17. https://doi.org/10.1186/s13005-020$\underline{00231-5}$

NAWROCKA, A. and LUKOMSKA-SZYMANSKA, M. The indirect bonding technique in Orthodontics: a narrative literature review. Materials (Basel, Switzerland). 2020, 13(4), 986. https://doi.org/10.3390/ma13040986

NOJIMA, L.I., ARAÚJO, A.S. and ALVES JÚNIOR, M. Indirect orthodontic bonding: a modified technique for improved efficiency and precision. Dental Press Journal of Orthodontics. 2015, 20(3), 109-117. https://doi.org/10.1590/2176-9451.20.3.109-117.sar

OLIVEIRA, N.S. and PRETTI, H. Digital technology in the refining of protocols for the bonding of orthodontic brackets. Journal of International Oral Health. 2018, 10(4), 168-171. https://doi.org/10.4103/jioh.jioh 14318

OLIVEIRA, N.S., et al. Comparison of the accuracy of virtual and direct bonding of orthodontic accessories. Dental Press Journal of Orthodontics. 2019, 24(4), 46-53. https://doi.org/10.1590/2177-6709.24.4.046-053.oar

PAMUKÇU, H. and ÖZSOY, Ö.P. Indirect bonding revisited. Turkish Journal of Orthodontics. 2016, 29(3), 80-86. https://doi.org/10.5152/TurkJOrthod.2016.16023

PAMUKÇU, H., ÖZSOY, Ö.P. and DAGALP, R. In vitro and in vivo comparison of orthodontic indirect bonding resins: a prospective study. Nigerian Journal of Clinical Practice. 2018, 21(5), 614-623. https://doi.org/10.4103/njcp.njcp 25217

PAUL, R.T., et al. Modified precision lingual bonding technique: a step-wise approach with torque angulation device-bracket positioning device. APOS Trends in Orthodontics. 2017, 7(4), 199-203. https://doi.org/10.4103/apos.apos 9716

READ, M.J.F. and O'BRIEN, K.D. A clinical trial of an indirect bonding technique with a visible light-cured adhesive. American Journal of Orthodontics and Dentofacial Orthopedics. 1990, 98(3), 259-262. https://doi.org/10.1016/S0889-5406(05)81603-8 
SHIMIZU, R.H., et al. Assessment of shear bond strength of brackets bonded by direct and indirect techniques: an in vitro study. Dental Press Journal of Orthodontics. 2012, 17(4), 23e1-23e7. https://doi.org/10.1590/\$2176-94512012000400008

SILVERMAN, E., et al. A universal direct bonding system for both metal and plastic brackets. American Journal of Orthodontics. 1972, 62(3), 236-244. https://doi.org/10.1016/s0002-9416(72)90264-3

SINGH, P., et al. Comparison of accuracy and time duration between direct and indirect bonding by photographic method: a short clinical study. International Journal of Contemporary Medical Research. 2019, 6(12), L14-L17. https://doi.org/10.21276/ijcmr.2019.6.12.19

SHARMA, N., et al. An in vivo comparative analysis of bond survival rate between two different bonding techniques. Journal of Dental Specialities. 2018, 6(2), 126-130. https://doi.org/10.18231/2393-9834.2018.0029

SONDHI, A. Effective and efficient indirect bonding: the Sondhi method. Seminars in Orthodontics. 2007, 13(1), 43-57.

https://doi.org/10.1053/j.sodo.2006.11.006

SPITZ, A., GRIBEL, B.F. and MARASSI, C. CAD/CAM Technology for digital indirect bonding. Journal of Clinical Orthodontics. 2018, 52(11), 621628.

TESSORE, E., MAZZOTTA, L. and FORTINI, A. Evaluation of shear bond strength and adhesive remnant index of orthodontic brackets bonded directly or indirectly with adhesive resin cements to bovine enamel. Journal of Dental Health, Oral Disorders and Therapy. 2017, 8(3), 521-526. https://doi.org/10.15406/idhodt.2017.08.00285

THOMAS, R.G. Indirect bonding: simplicity in action. Journal of Clinical Orthodontics. 1979, 13(2), 93-106.

WENDL, B., DROSCHL, H. and MUCHITSCH, P. Indirect bonding: a new transfer method. The European Journal of Orthodontics. 2008, 30(1), 100-107. https://doi.org/10.1093/ejo/cjm094

WHITE, L.W. A new and improved indirect bonding technique. Journal of Clinical Orthodontics. 1999, 33(1), 17-23.

ZANINI, M.M., et al. Periodontal conditions in orthodontic patients using direct and indirect bracket bonding techniques: a randomized study. Journal of Dentistry and Oral Hygiene. 2016, 8(9), 59-65. https://doi.org/10.5897/JDOH2015.0176

Received: 16 April 2021 | Accepted: 30 October 2021 | Published: 29 December 2021

This is an Open Access article distributed under the terms of the Creative Commons Attribution License, which permits unrestricted use, distribution, and reproduction in any medium, provided the original work is properly cited. 